\title{
Decentralized Control for Multichannel Active Vibration Isolation
}

\author{
Sang-Myeong Kim, Stephen J. Elliott, and Michael J. Brennan
}

\begin{abstract}
This paper describes a theoretical and experimental investigation into an active four-mount vibration isolation system, in which electromagnetic actuators are installed in parallel with each of the four passive mounts placed between a three-dimensional piece of equipment and a vibrating base structure. Decentralized velocity feedback control is applied, where each actuator is operated independently by feeding back the corresponding equipment vibration velocity at the same location. Although one end of the actuator acts at the sensor position on the equipment, the system is not collocated because of the reactive force at the other end acting on the flexible base structure, whose dynamics may be strongly coupled with the mounted equipment. The investigation of this actuator installation and its practical implementation are the motivation of this research. Isolation of low-frequency vibration is considered where the equipment can be modeled as a rigid body and the mounts as lumped-parameter springs and dampers. A general theoretical formulation for analysing multiple-mount vibration isolation systems using the impedance method is presented and is used to investigate the control mechanisms involved. Experimental results show that up to $14 \mathrm{~dB}$ reduction in the kinetic energy of the equipment can be achieved in practice. If very high gains are used in the experiments, however, instability occurs at low frequencies due to phase shifts in the transducer conditioning electronics.
\end{abstract}

Index Terms-Active isolation, decentralized control, velocity feedback, vibration control.

\section{INTRODUCTION}

A NTIVIBRATION mounts (also called isolators) are often used to protect delicate pieces of equipment from the vibration of the structure that they are attached to. With conventional passive mounts, however, there is a tradeoff between lowand high-frequency isolation performance, which depends on the damping in the mounts [1]. Thus, it is useful to introduce additional vibration sources that can actively reduce vibration transmission through the mounts at all frequencies [2], [3]. Although such active isolators may be constructed by feeding back all the state variables, as commonly seen in active suspension systems [4], [5], a simpler method may be to use direct output feedback control using the velocity responses of the structure to be isolated. When force actuators are placed in parallel with the isolators and they are collocated with the sensors then if the base structure behaves as a rigid body, the multichannel decentralized control system constructed by linking each collocated pair is passive and hence asymptotically stable [6], [7]. In this case, the control gains used can be analogously transformed to virtual mechanical skyhook dampers [8]. An experimental study

Manuscript received September 14, 1999; revised July 5, 2000 and August 28, 2000. Recommended by Guest Editors S.O. R. Moheimani and G. C. Goodwin S.-M. Kim is with the Department of Mechatronics, Kwang-Ju Institute of Science and Technology, Puk-gu 500-712, Korea (e-mail: ksm@kjist.ac.kr).

S. J. Elliott and M. J. Brennan are with the ISVR, University of Southampton, Southampton SO17 1BJ, U.K.

Publisher Item Identifier S 1063-6536(01)00420-1. using this idea has recently been reported with a two-mount active isolation system [9].

The work presented here describes a theoretical and experimental investigation into an active four-mount vibration isolation system in which electromagnetic actuators are installed in parallel with each of four passive mounts placed between a three-dimensional piece of equipment and a flexible vibrating base structure. Decentralized velocity feedback control is applied [10], [11], where each actuator is operated independently by simply feeding back a signal proportional to the corresponding equipment vibration velocity at the same location. Although one end of each actuator is collocated with each sensor on the equipment, the system is not collocated in the sense of [6], [7] because of the additional noncollocated reactive force at the other end acting on the flexible base structure which is also a part of the plant. When this is strongly coupled with the mounted equipment, the passivity property [6], [7] of collocated control is not strictly applicable. This practical configuration is investigated in the current paper, with particular emphasis being paid to the control mechanisms and stability. The impedance method [12] is used for the mechanical analysis of both passive and active vibration isolation systems. Isolation of low-frequency vibration is considered where the equipment can be modeled as a rigid body and the mounts as lumped parameter springs and dampers. The simple decentralized direct velocity feedback control system presented here could be applied to the active vibration isolation of delicate equipment, such as the instrument boxes in aeroplanes and telescopes in satellites, etc.

The basic concepts and mechanisms of velocity feedback control using the structural-borne actuator are discussed in Section II initially with a single-mount vibration isolation system. The analysis is extended to a general four-mount system in Section III, and experiment results are reported in Section IV. Potential causes of instability in practical implementations are also discussed, before the paper is concluded in Section V.

\section{Active Isolation of a Single Mount System}

Consider a single mount isolation system where a piece of equipment is connected to a flexible base structure via a single mount. If the mount is assumed to be massless, the whole system can be represented in terms of mechanical impedances as shown in Fig. 1(a) where $Z_{e}, Z_{m}$, and $Z_{b}$ denote the impedances of the equipment, mount, and base, respectively. The base structure with velocity $v_{b}$ is excited by a primary force $f_{p}$, and direct velocity feedback control is applied to reduce the equipment velocity $v_{e}$ using a pair of control forces $f_{c}$ via the controller with a gain $-H$. The force pair $f_{c}$ models the action and reaction of a single electromagnetic control actuator installed in parallel with 


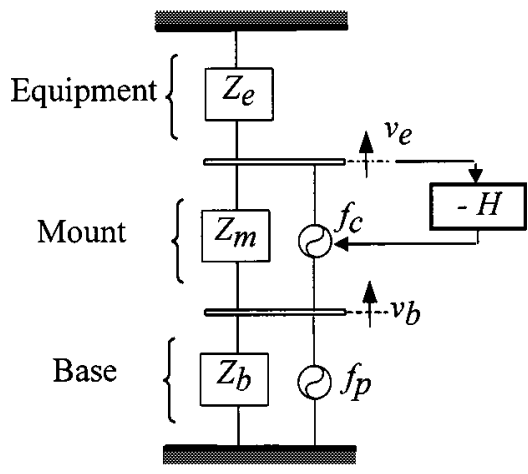

(a)

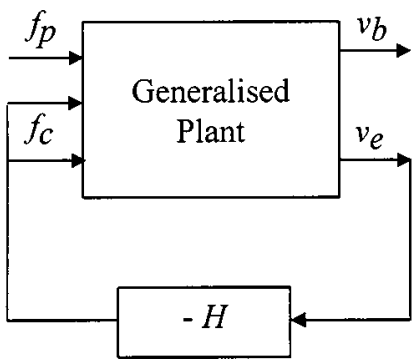

(b)

Fig. 1. (a) Impedence diagram and (b) block diagram of a single mount vibration isolation system.

the mount. It is convenient to interpret the mechanical system as a coupled one connecting the mounted equipment and the base structure, where the mounted equipment is the combination of the equipment and the mount. The impedance diagram can also be represented as a block diagram as shown in Fig. 1(b) where the generalized plant has three inputs and two outputs.

If the total force acting through the mount is $f_{m}$, then the dynamic equations of the mounted equipment can be written in the frequency domain as

$$
\begin{aligned}
Z_{e} v_{e} & =f_{m} \\
f_{m} & =f_{c}+Z_{m}\left(v_{b}-v_{e}\right)
\end{aligned}
$$

and the dynamics of the base structure can be described by

$$
Z_{b} v_{b}=f_{p}-f_{m}
$$

The system can also be represented in a matrix form as

$$
\left[\begin{array}{cc}
Z_{e}+Z_{m} & -Z_{m} \\
-Z_{m} & Z_{b}+Z_{m}
\end{array}\right]\left\{\begin{array}{l}
v_{e} \\
v_{b}
\end{array}\right\}=\left\{\begin{array}{c}
f_{c} \\
f_{p}-f_{c}
\end{array}\right\} .
$$

When direct velocity feedback control is applied, the control force is given by

$$
f_{c}=-H v_{e}
$$

where $H$ is restricted here to be a positive control gain. Incorporating (5) into (4) gives the active system written in a compact matrix form as

$$
\left[\begin{array}{cc}
Z_{e}+Z_{m}+H & -Z_{m} \\
-\left(Z_{m}+H\right) & Z_{b}+Z_{m}
\end{array}\right]\left\{\begin{array}{l}
v_{e} \\
v_{b}
\end{array}\right\}=\left\{\begin{array}{c}
0 \\
f_{p}
\end{array}\right\} .
$$

Note that, because of the $(2,1)$ term representing the influence of the reactive force acting on the base, the impedance matrix of the complete active system is nonsymmetric. This nonsymmetric term means that the stability of the system can not be assessed by simply using the definiteness property (positive or negative) of the impedance matrix as in collocated control [6], [7]. In addition, the mechanical analogy of a skyhook damper [8] is no longer a correct interpretation, as far as the dynamics of the complete coupled system is concerned. Here, the active system is temporarily assumed to be stable to investigating the control mechanisms involved, so that the responses $\left\{\begin{array}{ll}v_{e} & v_{b}\end{array}\right\}^{T}$ can be obtained simply by inverting the system impedance matrix.

The transmissibility is often used as a measure of control performance, which is given by [8]

$$
T=\frac{v_{e}}{v_{b}}=\frac{Z_{m}}{Z_{e}+Z_{m}+H} .
$$

Equation (7) suggests that, as far as the relative vibration of the equipment to the base $v_{e} / v_{b}$ is concerned, rather than $v_{e}$ or $v_{b}$ alone, the feedback control system acts like a skyhook damper. Note that the base impedance does not appear in (7). Transmissibility is a good performance measure for a mounted equipment that is weakly coupled to the base [12]. If strongly coupled, however, the base velocity $v_{b}$ changes after the attachment of the mounted equipment and also after the feedback controller is implemented.

If the feedback gain used is very large so that $H \gg\left|Z_{e}+Z_{m}\right|$ in (7), the transmissibility is greatly improved, i.e., $T \approx 0$. The control force in this case becomes

$$
f_{\mathrm{CO}} \approx-Z_{m} v_{b} \text {. }
$$

For an infinite gain this is the perfect control force for a hypothetical feedforward controller given perfect knowledge of $Z_{m}$ and $v_{b}$. If the control force $f_{c o}$ is applied, (6) becomes

$$
v_{e} \approx 0, \quad v_{b} \approx Y_{b} f_{p}
$$

where $Y_{b}=1 / Z_{b}$. With perfect control of equipment vibration the base would behave as if no mounted equipment were attached, showing that the control force pair uncouples the mounted equipment from the base. In practice this can only be achieved approximately.

We now examine stability of the single mount system shown as a generalized block diagram in Fig. 1(b). By inverting the passive impedance matrix in (4), the equipment velocity is

$$
v_{e}=G(j \omega) f_{c}+d_{e}
$$

where the frequency response of the plant $G(j \omega)$ is given by

$$
G(j \omega)=\frac{v_{e}}{f_{c}}=\frac{1}{Z_{e}+Z_{m}+Z_{m} Y_{b} Z_{e}}
$$

and the disturbance is $d_{e}=Y_{b} Z_{m} f_{p} /\left(Z_{e}+Z_{m}+Z_{m} Y_{b} Z_{e}\right)$ in which $Y_{b}=1 / Z_{b}$. If velocity feedback control is applied so that $f_{c}=-H v_{e}$, then the equipment velocity is

$$
v_{e}=\frac{d_{e}}{1+G(j \omega) H}
$$


which is the typical form for a disturbance rejection problem. Similarly, the base velocity after feedback can be obtained as

$$
v_{b}=\frac{Z_{e}+Z_{m}+H}{1+G(j \omega) H} \cdot \frac{d_{e}}{Z_{m}} .
$$

The control system is unconditionally stable with respect to the control gain $H$ if the plant response $G(j \omega)$ does not cross the negative real axis, i.e.,

$$
\operatorname{Re}(G(j \omega)) \geq 0 \text { or } 90^{\circ} \leq \angle G(j \omega) \leq 90^{\circ}
$$

at all frequencies. Here $\operatorname{Re}(\cdot)$ denotes the real part. Because of the passivity of the impedances of mechanical elements, i.e., $\operatorname{Re}\left(Z_{e}\right) \geq 0$ and $\operatorname{Re}\left(Z_{m}\right) \geq 0$, the condition in (14) is determined by the third term of the denominator $Z_{m} Z_{e} Y_{b}$ in (11). Thus the required stability condition can be rewritten as

$$
\operatorname{Re}\left(Z_{m} Z_{e} Y_{b}\right) \geq 0
$$

The equipment structure is now modeled as a free-free flexible beam of total mass $m_{e}$ and length $L$ that is installed on an arbitrary flexible base structure via a mount modeled as a single spring of impedance $Z_{m}=k_{m} / j \omega$ as shown in Fig. 2 . If we ignore all the flexible modes higher than the first, the equipment impedance is given by $Z_{e}=j \omega m_{e} \alpha(\omega)$, where $\alpha(\omega)=\left(\omega_{e}^{2}-\omega^{2}\right) /\left(\omega_{e}^{2}-\left(1+\phi^{2}\right) \omega^{2}\right)$ is a nondimensional variable [13]. Here $\omega_{e}$ is the first angular natural frequency of the flexible beam and $\phi^{2}$ is the squared value of the first mode shape function at the mount point, $L / 2$. For simplicity, damping in the equipment and the mount is ignored. It is important to note that at low frequencies where $\omega \leq \sqrt{\omega_{e}^{2} /\left(1+\phi^{2}\right)}, \alpha(\omega)$ is positive so that the equipment impedance is mass-like and behaves as a rigid body. Equation (15) in this case becomes

$$
\operatorname{Re}\left(m_{e} k_{m} \alpha(\omega) Y_{b}\right) \geq 0 .
$$

Since the real part of the base mobility $Y_{b}$ is always nonnegative, (16) is satisfied for $\omega \leq \sqrt{\omega_{e}^{2} / 1+\phi^{2}}$. If the equipment is completely rigid, i.e., $\alpha(\omega)=1$ so that $Z_{e}=j \omega m_{e}$, (16) becomes $m_{e} k_{m} \cdot \operatorname{Re}\left(Y_{b}\right) \geq 0$ and is valid at all frequencies. In this case, the Nyquist locus looks similar to the mobility of a single degree of freedom (DOF) vibration system, and is always in the right half of the complex plane. If a small amount of damping is included in the mount, the original locus shifts in the clockwise direction, but still approaches the origin as the frequency increases without crossing the negative real axis. An important conclusion from this analysis is that the system is unconditionally stable with respect to the control gain regardless of whether the actuator installation is grounded or not, provided the equipment is rigid and is supported upon a massless mount. It should be emphasized that no assumption has made about the base dynamics to draw this conclusion. Under these idealized conditions, the active isolation system shown in Fig. 1 has collocation control-like behavior (unconditionally stable and perfect controllability) and its plant shows driving point mobility-like behavior (no crossover of the negative real axis of the Nyquist plot).

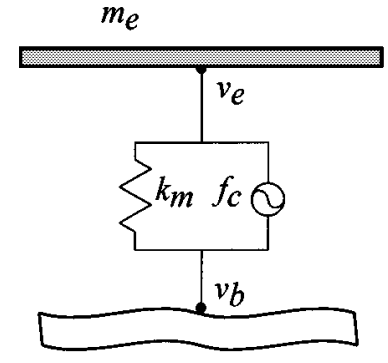

Fig. 2. One DOF equipment structure mounted on a flexible structure.

\section{ACtive Vibration Isolation of A Multiple-Mount SYSTEM}

Extending the single mount system to a four-mount system with a three-dimensional (3-D) piece of rigid equipment results in the system as shown in Fig. 3. The impedance approach described in the previous section can also be applied to this general case but with some care because of the difference between the numbers of mounts (four) and DOFs of the equipment structure (which is now assumed to move as a rigid body and so has only three DOFs). The coordinate transformations between the physical coordinates at the mounting points and modal coordinates are given by

$$
\mathbf{v}_{\mathbf{e}}=\mathbf{Q} \mathbf{a}, \quad \mathbf{a}=\mathbf{R} \mathbf{v}_{\mathbf{e}}
$$

where $\mathbf{a}=\{\dot{w} \dot{\theta} \dot{\phi}\}^{T}$ is the modal velocity vector consisting of the derivatives of heave, pitch, and roll motions at the mass centre, and $\mathbf{v}_{\mathbf{e}}=\left\{\begin{array}{llll}v_{e 1} & v_{e 2} & v_{e 3} & v_{e 4}\end{array}\right\}^{T}$ is the velocity vector at the mounting points. Since (17) is over-determined, the pseudoinverse of $\mathbf{Q}$ is give by $\mathbf{R}=\left(\mathbf{Q}^{\mathbf{T}} \mathbf{Q}\right)^{-1} \mathbf{Q}^{\mathbf{T}}$ [14], [15]. The dynamic equations can be described in a similar way to those for the single mount system given in (1)-(3). By extending (4), the system equations can be written as

$$
\left[\begin{array}{cc}
\overline{\mathbf{Z}}_{\mathbf{e}}+\overline{\mathbf{Z}}_{\mathbf{m}} & -\mathbf{Q}^{\mathrm{T}} \mathbf{Z}_{\mathbf{m}} \\
-\mathbf{Z}_{\mathbf{m}} \mathbf{Q} & \mathbf{Z}_{\mathbf{b}}+\mathbf{Z}_{\mathbf{m}}
\end{array}\right]\left\{\begin{array}{c}
\mathbf{a} \\
\mathbf{u}_{\mathbf{b}}
\end{array}\right\}=\left\{\begin{array}{c}
\mathrm{g}_{\mathbf{c}} \\
\mathbf{d}_{\mathbf{p}}-\mathrm{f}_{\mathbf{c}}
\end{array}\right\}
$$

where the modal impedance matrix of the equipment is $\overline{\mathbf{Z}}_{\mathbf{e}}=$ $j \omega \mathbf{J}_{\mathbf{e}}$ in which $\mathbf{J}_{\mathbf{e}}$ is the diagonal inertia matrix of the equipment whose diagonal terms are the mass of the equipment $M$, and the moment of inertia quantities to pitch and roll motions $I_{\theta}$ and $I_{\varphi}$. The bar notation is introduced to signify a matrix in the modal rather than the physical coordinates of the equipment. $\mathbf{Z}_{\mathbf{m}}$ is a $(4 \times 4)$ diagonal matrix whose diagonal terms are the impedances of each mount, $f_{c}$ is the control force vector $\mathbf{f}_{\mathbf{c}}=$ $\left\{\begin{array}{llll}f_{c 1} & f_{c 2} & f_{c 3} & f_{c 4}\end{array}\right\}^{T}, \mathbf{d}_{\mathbf{p}}=\mathbf{Z}_{\mathbf{b}} \mathbf{Y}_{\mathbf{b p}} \mathbf{f}_{\mathbf{p}}$ in which $\mathbf{Z}_{\mathbf{p}}$ and $\mathbf{Y}_{\mathbf{b p}}$ are the impedance and mobility matrices of the uncoupled base structure.

The generalized control force vector is $\mathbf{g}_{\mathbf{c}}=\mathbf{Q}^{\mathbf{T}} \mathbf{f}_{\mathbf{c}}$, and $\overline{\mathbf{Z}}_{\mathbf{m}}=\mathbf{Q}^{\mathbf{T}} \mathbf{Z}_{\mathbf{m}} \mathbf{Q}$ is the modal mount impedance matrix which is the transformed form of the physical matrix $\mathbf{Z}_{\mathbf{m}}$ to the equipment modal domain [14], [15]. When velocity feedback control with the control gain matrix $\mathbf{H}$ is applied, i.e., $\mathbf{f}_{\mathbf{c}}=-\mathbf{H v}_{\mathbf{e}}$, using the properties $\mathbf{g}_{\mathbf{c}}=\mathbf{Q}^{\mathbf{T}} \mathbf{f}_{\mathbf{c}}$ and $\mathbf{v}_{\mathbf{e}}=\mathbf{Q} \mathbf{a}$ gives the transformed gain matrix as

$$
\overline{\mathbf{H}}=\mathbf{Q}^{\mathbf{T}} \mathbf{H Q} .
$$




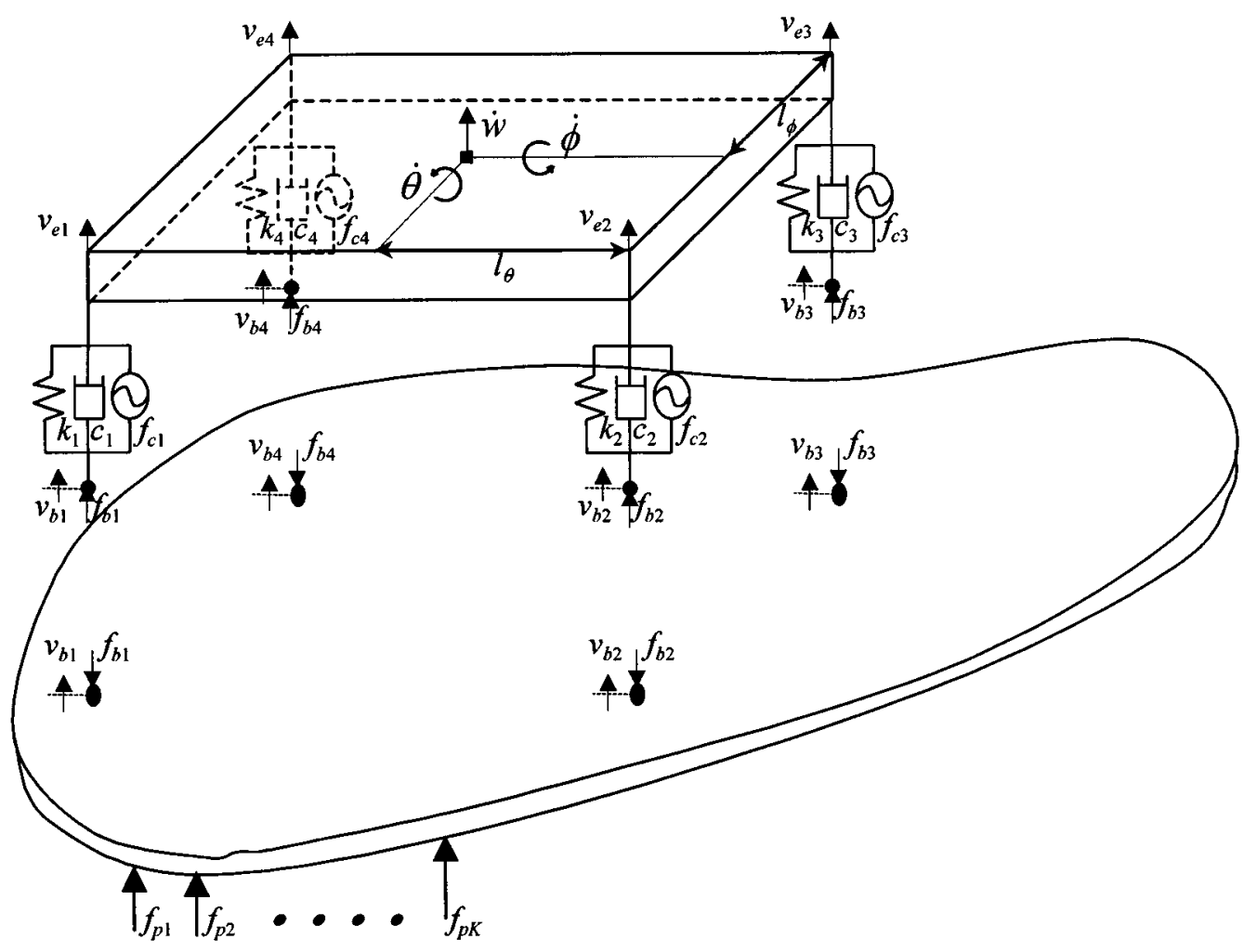

Fig. 3. A 3-D rigid equipment structure supported upon four mounts.

Again the controllers are restricted to be frequency independent constants. The complete dynamics of the active system can now be written as

$$
\left[\begin{array}{cc}
\overline{\mathbf{Z}}_{\mathbf{e}}+\overline{\mathbf{Z}}_{\mathbf{m}}+\overline{\mathbf{H}} & -\mathbf{Q}^{\mathbf{T}} \mathbf{Z}_{\mathbf{m}} \\
-\left(\mathbf{Z}_{\mathbf{m}}+\mathbf{H}\right) \mathbf{Q} & \mathbf{Z}_{\mathbf{b}}+\mathbf{Z}_{\mathbf{m}}
\end{array}\right]\left\{\begin{array}{c}
\mathbf{a} \\
\mathbf{u}_{\mathbf{b}}
\end{array}\right]=\left\{\begin{array}{c}
\mathbf{0} \\
\mathbf{d}_{\mathbf{p}}
\end{array}\right\} .
$$

Equation (21) is a compact description of the four-mount active vibration isolation system in terms of impedances. Note this is also a simple extension of the form given for the single mount system in (6). If the perfect control force vector $\mathbf{f}_{\mathbf{c o}}=-\mathbf{Z}_{\mathbf{m}} \mathbf{v}_{\mathbf{b}}$ is applied, it uncouples the mounted equipment from the vibrating base structure so that the active system becomes similar to (9). The solution of (21) can be obtained by inverting the impedance matrix, provided the system is stable. The kinetic energy for the 3-D equipment structure can be conveniently employed as a performance measure, and is given by

$$
E_{k}=\frac{1}{2} \mathbf{a}^{\mathbf{H}} \mathbf{J}_{\mathbf{e}} \mathbf{a}
$$

Consider a simplified control system where four separate singlechannel control systems are used by connecting each collocated pair of the actuators and sensors, instead of a general fourchannel control system with a fully populated gain matrix $\mathbf{H}$. This is termed decentralized control [10], [11] where each of the four actuators is controlled independently by feeding back the corresponding equipment vibration velocity response at the same location. In this case the control gain matrix $\mathbf{H}$ becomes diagonal. We investigate the active system shown in (21) when this simplified control strategy is applied. If the system shown in Fig. 3 is assumed to be statically balanced, which means the impedances of each mount are the same, $Z_{m}$, for a symmetric equipment structure, then $\overline{\mathbf{Z}}_{\mathbf{m}}$ becomes diagonal. If we further assume that the four gains of each controller are the same, $H$, the $(3 \times 3)$ matrix in the $(1,1)$ term of $(21)$ giving the equipment dynamics in modal coordinates also becomes a diagonal matrix whose diagonal terms are $j \omega M+4\left(Z_{m}+H\right), j \omega J_{\theta}+$ $4\left(Z_{m}+H\right) l_{\theta}^{2}$ and $j \omega J_{\phi}+4\left(Z_{m}+H\right) l_{\phi}^{2}$. Here $l_{\theta}$ is the distance between the mass centre of the equipment and the mount in pitch motion, and $l_{\phi}$ in roll motion can be similarly defined. Note, as in the single mount case, the control gain $H$ is simply added to the mount impedance $Z_{m}$ in all three modal motions. For the statically balanced symmetric structure shown in Fig. 3, decentralized control using the same gains (equidecentralized control) thus results in a modal controller, which equally damps all modes (equimodal control). Each of the four gains acts like a skyhook damper, as far as the relative equipment vibrations are concerned as discussed in Section II. The analysis can now be extended to the general case of a nonsymmetric equipment structure, but whose installation is again assumed to be statically balanced as is preferred in practice [1], in which case equimodal control can again be achieved by making the modal control gain matrix $\overline{\mathbf{H}}$ diagonal, and similar to $\overline{\mathbf{Z}}_{\mathbf{m}}$.

We now examine stability of the decentralized control system. The following analysis for stability is very similar to that for the single mount case. The multichannel plant denoted as $\mathbf{G}(j \omega)$ is now a $(4 \times 4)$ matrix satisfying $\mathbf{v}_{\mathbf{e}}=\mathbf{G}(j \omega) \mathbf{f}_{\mathbf{c}}$. This matrix is nonsymmetric in general due to the nonsymmetric installation of the mounted equipment on the base, and can be obtained by solving (19) with $\mathbf{d}_{\mathbf{p}}=\mathbf{0}$ and using the transformation relation in (17). Stability of the multichannel system can be determined 
from the open-loop frequency response function matrix written as $\mathbf{L}(j \omega)=\mathbf{G}(j \omega) \mathbf{H}$. The generalized Nyquist criterion states that the closed-loop system is stable if and only if none of the eigenvalue loci of $\mathbf{L}(j \omega)$ should encircle the $(-1,0)$ point in the complex plane [10]. More specifically for equidecentralized control, i.e., $\mathbf{H}=H \mathbf{I}$, then $\mathbf{L}(j \omega)=H \mathbf{G}(j \omega)$, and none of the eigenvalue loci of $\mathbf{G}(j \omega)$ should encircle the $(-1,0)$ point for stability, and none of the eigenvalue loci should cross the negative real axis for unconditional stability. Since one of the responses in $\mathbf{v}_{\mathbf{e}}$ is linearly dependent on the others and thus one of the eigenvalues is zero, it is convenient to judge the criterion in the transformed coordinates

$$
\operatorname{eig}(\mathbf{R G}(j \omega) \mathbf{Q})
$$

where $\operatorname{eig}(\cdot)$ denotes the eigenvalues.

Now we examine a particular case when the coordinate transformations are fully determined, i.e., $\mathbf{Q}$ in (17) is square and invertible, such as the two mount system with a two-dimensional equipment and the three mount system with a three-dimensional equipment. In this case, analysis of the stability condition given in (23) is greatly simplified. The transform matrix in (18) is now can be written as $\mathbf{R}=\mathbf{Q}^{-1}$, and the same coordinate transform relations can also be applied to the base velocity vector as $\mathbf{v}_{\mathbf{b}}=\mathbf{Q b}$ and $\mathbf{b}=\mathbf{R} \mathbf{v}_{\mathbf{b}}$, where $\mathbf{b}$ is the transformed base velocity vector. In this case the plant matrix is given by

$$
\mathbf{G}(j \omega)=\mathbf{Q}\left(\overline{\mathbf{Z}}_{\mathbf{e}}+\overline{\mathbf{Z}}_{\mathbf{m}}+\overline{\mathbf{Z}}_{\mathbf{m}} \overline{\mathbf{Y}}_{\mathbf{b}} \overline{\mathbf{Z}}_{\mathbf{e}}\right)^{-1} \mathbf{Q}^{\mathbf{T}}
$$

where $\overline{\mathbf{Y}}_{\mathbf{b}}=\overline{\mathbf{Z}}_{\mathbf{b}}^{-1}$, in which the transformed base impedance is $\overline{\mathbf{Z}}_{\mathbf{b}}=\mathbf{Q}^{\mathbf{T}} \mathbf{Z}_{\mathbf{b}} \mathbf{Q}$. Note that this is also a simple extension of the form for the single mount system in (11). Again a rigid equipment and massless mounts are assumed together with the statically balanced installation of a symmetric equipment. Note, however, no assumption has made on the base dynamics. In a similar way to the single mount case given in (14), a satisfactory condition for unconditional stability using (23) can be written as

$$
\operatorname{Re}\left(\operatorname{eig}\left(\left(\overline{\mathbf{Z}}_{\mathbf{e}}+\overline{\mathbf{Z}}_{\mathbf{m}}+\overline{\mathbf{Z}}_{\mathbf{m}} \overline{\mathbf{Y}}_{\mathbf{b}} \overline{\mathbf{Z}}_{\mathbf{e}}\right)^{-1}\right)\right) \geq 0
$$

at all frequencies. Here $\overline{\mathbf{Z}}_{\mathbf{e}}$, and $\overline{\mathbf{Z}}_{\mathbf{m}}$ are diagonal, and $\overline{\mathbf{Y}}_{\mathbf{b}}$ is symmetric but nondiagonal. The condition can be determined by examining eig $\left(\overline{\mathbf{Z}}_{\mathbf{e}}+\overline{\mathbf{Z}}_{\mathbf{m}}+\overline{\mathbf{Z}}_{\mathbf{m}} \overline{\mathbf{Y}}_{\mathbf{b}} \overline{\mathbf{Z}}_{\mathbf{e}}\right)$. Because of passivity, then $\operatorname{Re}\left(\operatorname{eig}\left(\overline{\mathbf{Z}}_{\mathbf{e}}\right)\right) \geq 0$ and $\operatorname{Re}\left(\operatorname{eig}\left(\overline{\mathbf{Z}}_{\mathbf{m}}\right)\right) \geq 0$, and the stability of the system is determined by the last term $\operatorname{eig}\left(\overline{\mathbf{Z}}_{\mathbf{m}} \overline{\mathbf{Y}}_{\mathbf{b}} \overline{\mathbf{Z}}_{\mathbf{e}}\right)$, where $\operatorname{Re}\left(\operatorname{eig}\left(\overline{\mathbf{Y}}_{\mathbf{b}}\right)\right) \geq 0$ again due to the passivity. If we assume that there is no damping in the mounts, then the diagonal matrix $\overline{\mathbf{Z}}_{\mathbf{m}} \overline{\mathbf{Z}}_{\mathbf{e}}$ has elements which are positive real and similar to that for the single mount system. Thus $\operatorname{Re}\left(\operatorname{eig}\left(\overline{\mathbf{Z}}_{\mathbf{m}} \overline{\mathbf{Y}}_{\mathbf{b}} \overline{\mathbf{Z}}_{\mathbf{e}}\right)\right) \geq 0$, and consequently the real eigenvalues of the plant in (25) become nonnegative at all frequencies so that $\operatorname{Re}(\operatorname{eig}(\mathbf{R G}(j \omega) \mathbf{Q})) \geq 0$. As in the case of the single mount system, damping in the mounts does not threaten the unconditional stability. However, if the equipment structure is flexible within the frequency range of interest, then $\operatorname{Re}\left(\operatorname{eig}\left(\overline{\mathbf{Z}}_{\mathbf{m}} \overline{\mathbf{Z}}_{\mathbf{e}}\right)\right)$ is no longer positive. Thus the decentralized control system is only unconditionally stable, provided the equipment behaves as a rigid body and the mounts act as springs and dampers.
When the system is over-determined as in the four-mount system in Fig. 3, there is a redundant passive mount and a redundant active force. It is thus no longer possible to apply the convenient transforms $\mathbf{v}_{\mathbf{b}}=\mathbf{Q b}$ and $\mathbf{b}=\mathbf{R v}_{\mathbf{b}}$ for the base responses. The analytical plant matrix expression may still be obtained from using (19), but becomes very complex in this case. Its analytical proof is thus not given but instead some supporting simulation and experimental results are reported in the next section.

\section{EXPERIMENTAL IMPLEMENTATION}

\section{A. Control Performances}

Decentralized direct velocity control was experimentally implemented with a statically balanced symmetric four-mount system similar to that shown in Fig. 3. The experimental setup is shown in Fig. 4(a) where a symmetrical aluminum block $(300 \times 160 \times 20 \mathrm{~mm})$ representing the equipment is installed on top of a free-free-clamped-clamped steel base plate of $(700 \times 500 \times 2 \mathrm{~mm})$ via four identical mounts, each of stiffness $k_{m}=4.2 \times 10^{4} \mathrm{~N} / \mathrm{m}$ and damping $c_{m}=25.6 \mathrm{Ns} / \mathrm{m}$. To realize the clamped-clamped boundary conditions, two opposite sides of the base plate were bolted on stiff frames. The large shaker underneath the plate acted as the primary force actuator, and the four small electromagnetic actuators fixed on the thick equipment plate were the control actuators at each mount position. The equipment to be isolated was thus a combined structure of the four actuators and the aluminum block, of thickness 20 $\mathrm{mm}$, whose inertial values were $M_{s}=6.232 \mathrm{~kg}, I_{\theta}=0.0685$ $\mathrm{kgm}^{2}, I_{\phi}=0.0162 \mathrm{kgm}^{2}$. Fig. 4(b) shows the structure of decentralized control for a single channel. Each actuator was fixed on top of the thick equipment plate at each mount location, and a stinger was connected between the actuator and the mount foot through the hole of the cylindrical mount, which was made from natural rubber. The equipment acceleration at the mounting point $\dot{v}_{e}$ was measured and passed through an integrator to obtain the velocity response $v_{e}$, which was then fed back to the actuator via a power amplifier with gain control. The integrator was contained in a commercial charge amplifier (B\&K type 2635) which also had high- and low-pass filter modules. The highpass filter cutoff frequency was set to be 1 $\mathrm{Hz}$ to avoid DC signal overflow, and the low-pass filter cutoff frequency was set to be $1 \mathrm{kHz}$ to filter out any measurement of flexible modes within the equipment structure. Only isolation of low-frequency vibration under $200 \mathrm{~Hz}$ was considered, so that the equipment structure could be modeled as a rigid body and each mount as a parallel connection of spring $k_{m}$ and damper $c_{m}$, as shown in Fig. 3 .

To measure the plant response, the actuator input voltage $V_{\text {in }}$ in Fig. 4(b) was used as the input signal instead of the input force because the input voltage at low frequencies is approximately proportional to the force input [16]. The measured plant responses were used to construct the complete plant matrix $\mathbf{G}(j \omega)$ in (24). Stability was assessed by applying the generalized Nyquist criterion as discussed earlier. The resulting eigenvalue loci are shown in Fig. 5(a), (b) for the theoretical and experimental models, respectively. The solid line denotes the locus of the heave mode, and dashed and dotted lines 


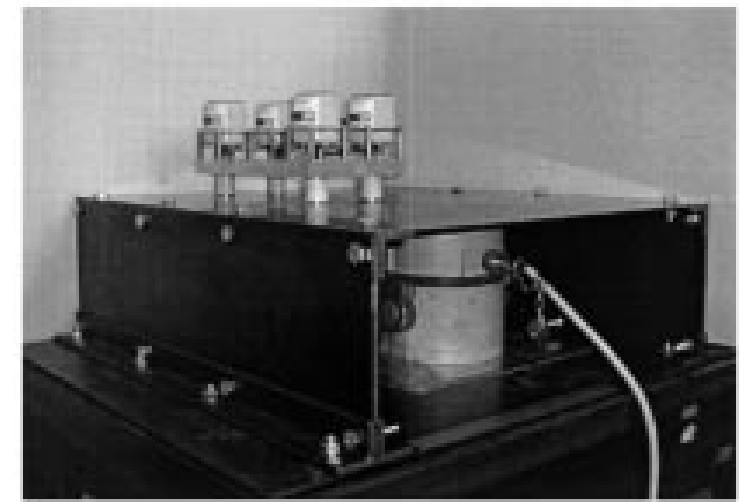

(a)

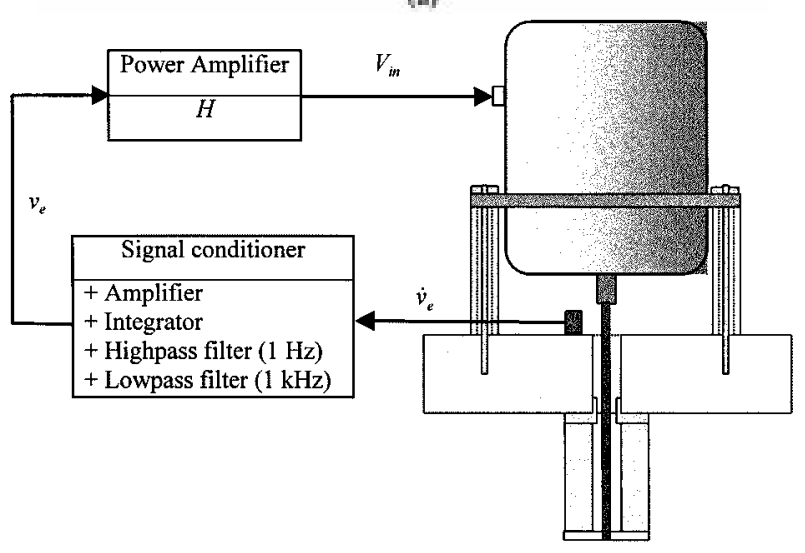

(b)

Fig. 4. (a) Experimental arrangement in which an aluminum block, to which the secondary actuators are attached, is supported on a steel plate by four active mounts and the steel plate can be vibrated with a large shaker. (b) The structure of a single active mount.

denote the loci of the pitch and roll modes, respectively. The experimental loci in Fig. 5(b) are very similar to the theoretical ones in Fig. 5(a), but it is difficult to judge whether or not they really cross the negative real axis near the origin, especially at frequencies less than $5 \mathrm{~Hz}$, where the data was of low coherence due to the low sensitivity of the actuators and sensors used. Zooming into the origin of the simulation plot showed that none of the eigenvalue loci cross the negative real axis, which is evidence that the redundancy of one actuator in the four-mount system does not threaten stability. Although not shown here, the use of three actuators was also examined both theoretically and experimentally and showed a similar result [13].

The four-channel decentralized control system whose single channel structure is shown in Fig. 4(b) was then tested. The control performance was measured with four equal gains $H$ in the power amplifiers and compared with simulations. The equipment velocity at the mounting points was measured and then transformed to modal responses using (18) to calculate the total kinetic energies in (22), which was used to quantify the control performance. The total kinetic energy calculated from the simulation model and measured in the experiment is shown in Fig. 6(a) and (b), respectively. In Fig. 6, the response before control is shown with solid lines and those after control are shown with dashed lines. The highest response (solid line) is the total kinetic energy before control, and the others (dashed

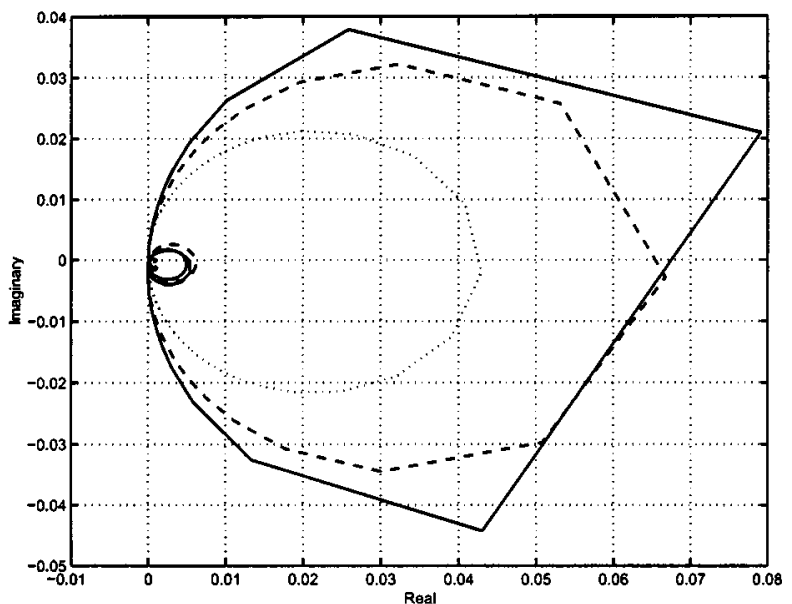

(a)

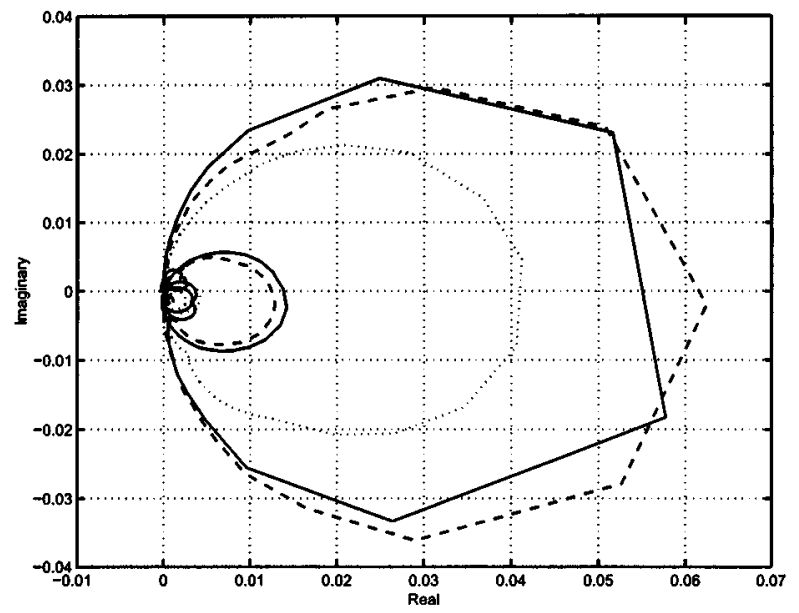

(b)

Fig. 5. (a) Predicted and (b) measured Eigenvalue loci of the plant on the flexible base structure; heave (solid), pitch (dashed), and roll (dotted).

lines) correspond to gains of 110,320 , and 1000. Higher gains could not be applied to the experimental plant because an instability occurred at about $1 \mathrm{~Hz}$, which was due to the cutoff frequency of the high-pass filter used in conjunction with the integrator. The reason for the instability was found to be phase advances at very low frequencies in the commercial power amplifier and integrator, which is discussed in more detail in the next section. Close inspection of the frequency range below 5 $\mathrm{Hz}$ in Fig. 6(b) reveals that the velocity with active control exceeds the uncontrolled value. However, the experimental results above $5 \mathrm{~Hz}$ agree well with the simulations shown in Fig. 6(a). Since the theoretical model implicitly assumes that the power amplifier and the integrator are ideal, it is unconditionally stable so that the results of using the higher gains of 3350 and 10000 are also shown in Fig. 6(a). It can be seen that, as the gain increases, the total energy is reduced over the whole frequency range in the simulations, without any amplification. The control performance of both the simulations and the experiments are tabulated in Table I according to the gain values used. The gains $H$ were transformed to skyhook damping ratios calculated based on the heave mode. The passive damping ratios of each mode were about $5 \%$. The total kinetic energy of the experimental equipment was calculated from $5 \mathrm{~Hz}$ upwards to avoid 


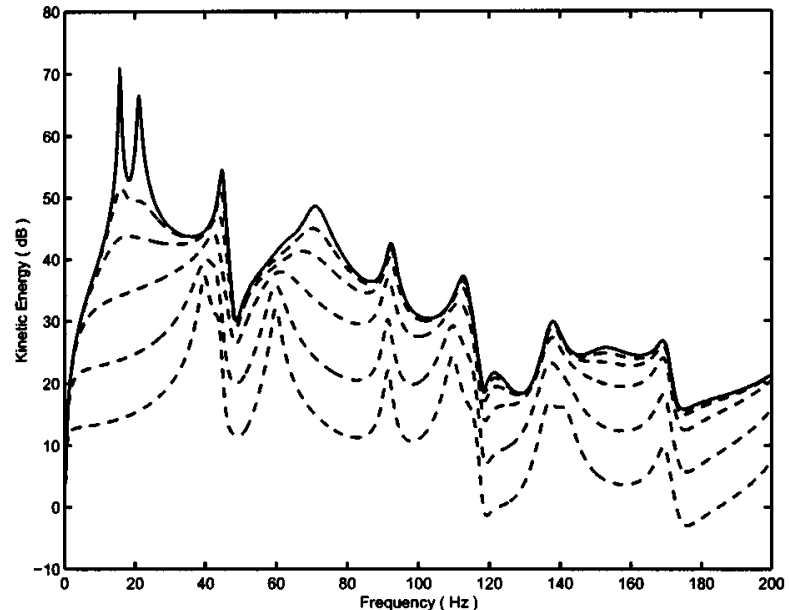

(a)

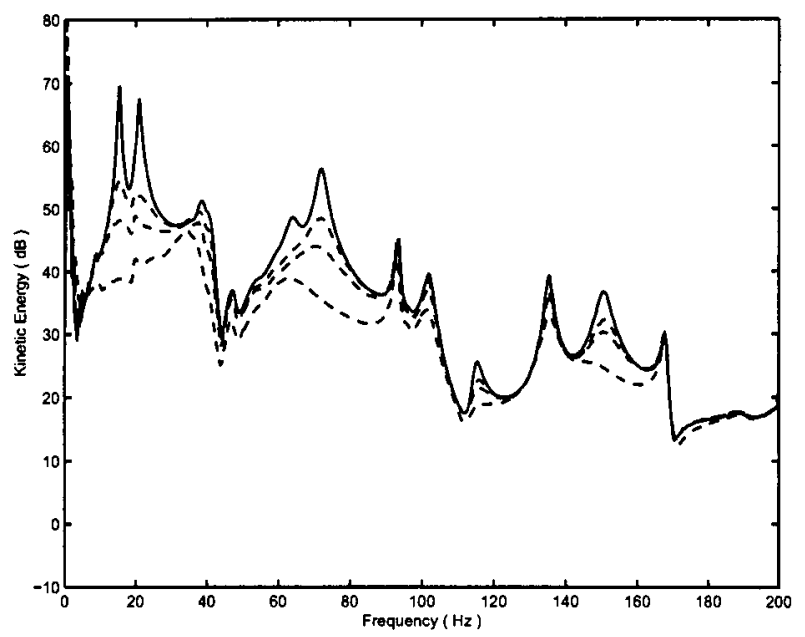

(b)

Fig. 6. (a) Predicted and (b) measured total kinetic energies of the equipment structure with various feedback gains $H$; without control (solid) and with control (dashed). (dB ref. $=10^{-10} \mathrm{~J}$ )

accounting for the amplification region. Both simulation and experiment results show a kinetic energy reduction of more than $14 \mathrm{~dB}$ for a feedback gain 1000 .

In order to explore the control mechanisms discussed above, a base velocity was also calculated and measured during control. The simulation and experimental results are shown in Fig. 7(a) and (b), respectively. As the gain increases, the original base response (solid line) approaches the uncoupled base response (dotted line), that is the base response without the mounted equipment attached. The dashed lines correspond to the base responses for each control gain. These results clearly demonstrate that the active isolation system tends to uncouple the mounted equipment from the base structure.

\section{B. Causes of Instability in the Practical System}

The cause of the instability in the experimental plant was found to be phase advances in the power amplifier and the integrator [13]. The commercial amplifier had a phase advance up to about $90^{\circ}$ at very low frequencies (under $5 \mathrm{~Hz}$ ). Furthermore, an additional phase advance occurred in the commercial charge amplifier used for integration. A phase advance of greater than
TABLE I

COMPARISON OF THE CONTROL PERFORMANCES, WHERE $c_{m}=25.6 \mathrm{Ns} / \mathrm{ms} \mathrm{IS}$ the Damping Value of the Passive Mounts

\begin{tabular}{c|c|c|cc}
\hline \multicolumn{3}{c|}{ Gains } & \multicolumn{2}{c}{ Overall reduction(dB) } \\
\hline \hline Absolute & Skyhook & Skyhook & & \\
Value, $H$ & Damping & Damping Ratio & Theory & Experiment \\
\hline 110 & $4.3 \times c_{m}$ & 0.215 & -7.3 & -7.1 \\
320 & $12.5 \times c_{m}$ & 0.626 & -10.9 & -9.9 \\
1000 & $39.1 \times c_{m}$ & 1.955 & -15.0 & -14.0 \\
3350 & $130.9 \times c_{m}$ & 6.548 & -18.2 & $\cdot$ \\
10000 & $390.6 \times c_{m}$ & 19.546 & -20.9 &. \\
\hline
\end{tabular}

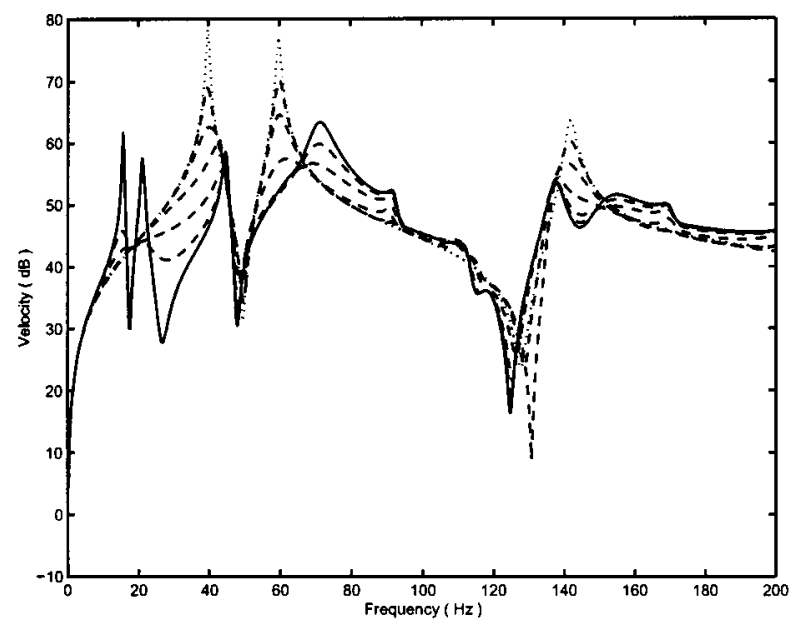

(a)

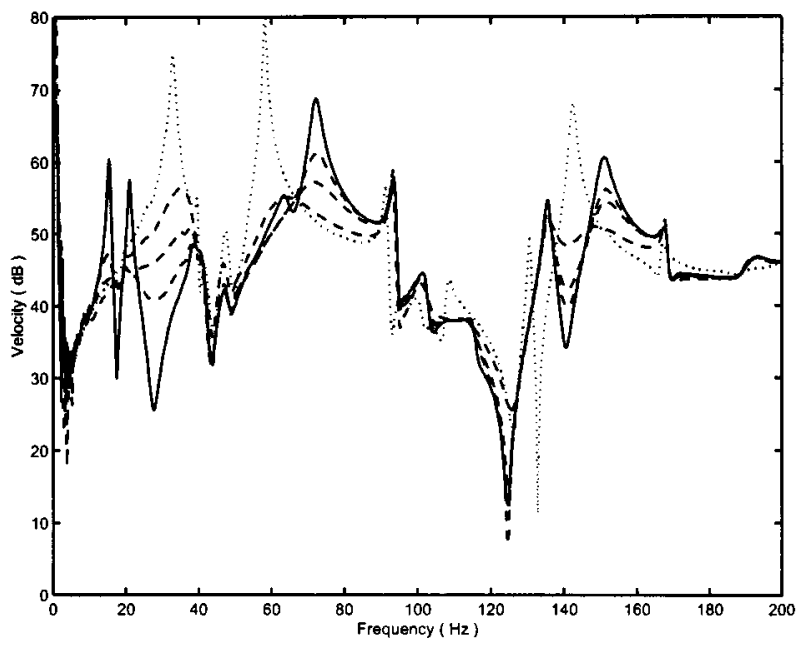

(b)

Fig. 7. (a) Predicted and (b) measured base response at the bottom of mount 1 for various feedback gains $H$; without control but coupled (solid), uncoupled (dotted), and with control (dashed).

$90^{\circ}$ at very low frequencies can cause the eigenvalue loci to cross the negative real axis, and thus make the system unstable to a high gain. Such phase advances, which were not accounted for in the simulations, were the cause of the instability in the experiment.

The experimental plant can also be potentially unstable at very high frequencies due to both electrical and mechanical causes. Use of an electrical low-pass filter incorporated inside 
the signal conditioner causes an effective time delay on the control loop, which can make the system unstable at very high frequencies [13]. Furthermore, the phase shift in the electromagnetic actuators can also be modeled as an additional time delay [16]. In the experimental plant considered, the equivalent delays were measured to be approximately $0.2 \mathrm{~ms}$ for the filter and 0.04 $\mathrm{ms}$ for the actuator, but the magnitude of the plant response had fallen off sufficiently at frequencies where these delays caused significant phase shift that the stability was not compromised. The system can also be unstable due to mechanical causes, particularly when the flexible modes of the equipment becomes important and when the mass of the mounts becomes significant. In the experimental plant, the first flexible mode of the equipment plate was at about $1240 \mathrm{~Hz}$, and the first longitudinal mode of the rubber mount was at about $500 \mathrm{~Hz}$ [13], which were both well above the frequencies at which good active performance would be expected in this application.

\section{CONCLUSION}

This paper has investigated both theoretically and experimentally a four-mount active vibration isolation system where electromagnetic actuators are installed in parallel with each of four mounts placed between a piece of equipment and a vibrating base structure. Decentralized velocity feedback control was used to actively achieve low-frequency vibration isolation.

A general formulation using the impedance approach was presented to analyze passive and active multiple isolation systems. This facilitated both an understanding the physics of vibration isolation and investigations into the control mechanisms involved. It was demonstrated that the decentralized velocity feedback controller tends to uncouple the mounted equipment from the base structure. It was shown that the system is unconditionally stable with respect to the control gain provided that the equipment is rigid and the mass effect of the mounts is negligible. Experimental results show that up to $14 \mathrm{~dB}$ reduction in the kinetic energy of the equipment can be achieved in practice. When very high gains were used, however, instability was encountered in the experimental rig at about $1 \mathrm{~Hz}$ due to undesirable phase advances of over than $90^{\circ}$ in the electrical equipment used.
Although the performance of the control system could be improved by implementing a fully coupled feedback system and by using a frequency dependent controller, the decentralized control system with constant feedback gains is very simple to implement and performs well in this application. It also has the advantage that very few assumptions have to be made about the dynamics of the system under control, and so the stability and performance of the control system is very robust to the kind of changes which may occur in these dynamics under operating conditions.

\section{REFERENCES}

[1] C. E. Crede and J. E. Ruzicka, "Theory of vibration isolation," in Shock and Vibration Handbook, C. M. Harris, Ed. New York: McGraw-Hill, 1996, ch. 30.

[2] L. Meirovitch, Dynamics and Control of Structures. New York: Wiley, 1990.

[3] C. R. Fuller, S. J. Elliott, and P. A. Nelson, Active Control of Vibration. New York: Academic, 1996.

[4] D. Hrovat and M. Hubbard, "Optimal vehicle suspensions minimizing RMS rattlespace, sprung-mass acceleration and jerk," J. Dyn. Syst., Measurement, Contr., vol. 103, pp. 228-236, 1981.

[5] A. Hac, "Stochastic optimal control of vehicles with elastic body and active suspension," J. Dyn. Syst., Measurement, Contr., vol. 108, pp. 106-110, 1986

[6] M. J. Balas, "Direct velocity feedback control of large space structures," J. Guidance Contr, vol. 2, pp. 252-253, 1979.

[7] S. M. Joshi, "Robustness properties of collocated controllers for flexible spacecraft," J. Guidance Contr., vol. 9, pp. 85-91, 1986.

[8] D. Karnopp, M. J. Crosby, and R. A. Harwood, "Vibration control using the semiactive force generators," J. Eng. Ind., pp. 619-626, 1974.

[9] M. Serrand, "Active Isolation of Base Vibration," M.Sc. thesis, Univ. Southampton, 1998.

[10] S. Skogestad and I. Postlethwaite, Multivariable Feedback Control; Analysis and Design: Wiley, 1996.

[11] M. Morari and E. Zafiriou, Robust Process Control. Englewood Cliffs, NJ: Prentice-Hall, 1989.

[12] S. M. Kim and M. J. Brennan, "A compact matrix formulation using the impedance and mobility approach for the analysis of structural-acoustic systems," J. Sound Vibr., no. 223, pp. 97-113, 1999.

[13] S. M. Kim, S. J. Elliott, and M. J. Brennan, "Active vibration isolation of a 3-D structure using velocity feedback control,", ISVR Tech. Memo. $845,1999$.

[14] B. Noble and J. W. Daniel, Applied Linear Algebra. Englewood Cliffs, NJ: Prentice-Hall, 1988.

[15] F. B. Hilderbrand, Methods of Applied Mathematics. Englewood Cliffs, NJ: Prentice-Hall, 1965.

[16] M. Z. Ren, K. Seto, and F. Doi, "Feedback structural-borne sound control of a flexible plate with an electromagnetic actuator: The phase lag problem,” J. Sound Vibr., vol. 205, pp. 57-80, 1997. 